\title{
HIV, Hepatitis B and C among people who inject drugs: high prevalence of HIV and Hepatitis C RNA positive infections observed in Delhi, India
}

Lopamudra Ray Saraswati ${ }^{1}$, Avina Sarna ${ }^{1 *}$, Mary Philip Sebastian¹, Vartika Sharma ${ }^{1}$, Ira Madan², Ibou Thior ${ }^{3}$, Julie Pulerwitz ${ }^{3,4}$ and Waimar Tun ${ }^{4}$

\begin{abstract}
Background: India has large PWID (persons who inject drugs) population estimated at 177,000. PWIDs are at high risk for HIV, Hepatitis B (HBV) and Hepatitis C (HCV) infections. We report the prevalence of HIV, HBV and HCV infections and correlates of HIV-HCV co-infection among male PWIDs in Delhi.

Methods: 3748 male PWIDs were recruited for a longitudinal HIV incidence study. Participants were tested for HBV and HCV infections at their first follow-up visit (FV1) using serum HBV-surface antigen, and HCV-antibody tests followed by HCV RNA PCR, respectively. All PWIDs who were HIV-negative at enrollment, were re-tested for HIV at FV1. Multinomial logistic regression was employed to identify predictors of HIV, HCV and HIV-HCV co-infection.

Results: Overall prevalence of HIV, HBV and HCV among 2,292 participants tested at FV1 was $25.9 \%, 9.7 \%$ and $53.7 \%$, respectively. $6.4 \%$ of the participants had HIV mono-infection, $34.1 \%$ had HCV mono-infection, and $19.6 \%$ had HIV-HCV co-infection. $26 \%$ of HIV-positive participants without HCV were HBsAg positive.

In the regression model, having practiced at least one risky injection in the past month (relative risk ratio (RRR): 1.38; $95 \%$ Cl: 1.01-1.89) and not knowing his own HIV status (RRR: 1.65, $95 \%$ Cl: 1.25-2.17) were independent predictors for HIV-HCV co-infection. Longer duration of drug injections was associated with a higher likelihood of HCV mono-infection (2-5 years RRR: $2.13 ; 6-10$ years RRR: 2.74; $\geq 11$ years RRR: 3.14 ) and HIV-HCV co-infection (2-5 years RRR: $5.14 ; 6-10$ years RRR: 8.53 ; $>11$ years RRR: 8.03). Higher frequency of injection days/month was associated with a higher likelihood of HCV mono-infection ( $\leq 10$ days/month RRR: $1.61 ; 11-20$ days/month RRR: 3.15; $21-30$ days/month RRR: 3.47 ) and HIV-HCV co-infections ( $\leq 10$ days/month RRR: 2.26; 11-20 days/month RRR: 3.46; $21-30$ days/month RRR: 4.83).
\end{abstract}

Conclusions: We report a high prevalence of HIV, HCV and HIV-HCV co-infection among male PWIDs in Delhi. A tenth of the participants were HBsAg positive. Targeted Intervention programs should make HBV/HCV testing, prevention and care more accessible for PWIDs.

Keywords: HIV, Hepatitis B, Hepatitis C, HIV-HCV co-infection, People Who Inject Drugs (PWID), India

\footnotetext{
*Correspondence: asarna@popcouncil.org

${ }^{1}$ Population Council, Zone 5A, India Habitat Centre, New Delhi 110003, India

Full list of author information is available at the end of the article
} 


\section{Background}

People who inject drugs (PWIDs) are at high risk for blood-borne infections that include HIV, hepatitis B (HBV) and $\mathrm{C}$ virus (HCV). $\mathrm{HCV}$ has been identified as the most common viral infection affecting PWIDs [1] and $\mathrm{HCV}$ is estimated to be 10 times more infectious than HIV, per unit of blood required for transmission [1]. Chronic HCV infection is associated with chronic liver disease including cirrhosis and end-stage liver disease [2, 3]; about $80 \%$ of individuals exposed to $\mathrm{HCV}$ develop chronic infection and 3-11\% with chronic HCV develop liver cirrhosis within 20 years [4]. HIV and HCV co-infection adversely affect the course and prognosis of both conditions [5-9]. In the case of HBV, $5 \%$ of adults exposed to the virus develop chronic HBV infection, and cirrhosis and death because of hepatocellular carcinoma are important complications of chronic HBV infection [10, 11].

The HIV epidemic in India is concentrated among sex workers, people who inject drugs (PWIDs), and men who have sex with men - often called 'most-at-risk-populations' (MARPs) - and among these groups PWIDs have the second highest HIV prevalence in the country (7.14 \%) [12]. India has an estimated 177,000 PWIDs [12]. The PWID population has been largely studied in the high HIV prevalence states in north-eastern and southern parts of the country, where HIV (25.4 -59.6\%), HBV (10 \%) and HCV (54.5-90.4 \%) prevalence has been reported [9,13-15]. Although there is emerging evidence of PWID presence in low HIV prevalence states of the country (the north-western part), only a few studies have documented HIV (29-37 \%), HBV (40 \%) and HCV (36-49 \%) prevalence in these states [16-18]. The PWID populations in the northeastern, southern, and western parts of the country differ significantly on sociodemographic characteristics, drug use patterns, and awareness of HIV and hepatitis B and C infections [19] highlighting the need for more epidemiological evidence on the prevalence and correlates of hepatitis in the PWID population. In this paper we report on the prevalence of HIV and Hepatitis B and C infections in a large cohort of male PWIDs from Delhi. Further we characterize the correlates of HIV and Hepatitis C co-infection in this population.

\section{Methods}

\section{Study setting}

The Population Council, in collaboration with PATH's Arise - Enhancing HIV Prevention Programs for At-Risk Populations, initiated a longitudinal cohort study, at five Drop-in Centers (DICs) run by Sahara Centre for Rehabilitation and Residential Care in Delhi, to examine HIV incidence and behavior change among PWIDs, before and after the provision of comprehensive HIV prevention interventions that follow WHO/UNAIDS/UNODC guidelines.
The study entailed three rounds of data collection: baseline, follow-up visit 1 (FV1) and follow-up visit 2 (FV2); the median follow-up time between baseline and FV1 was 9.8 months (IQR: 8.2-11.1 months), and between FV1 and FV2 was 12.0 months (IQR: 10.7-13.2 months). HIV testing was undertaken at baseline to determine the HIV status of the cohort [20]. At FV1, all participants underwent testing for hepatitis B and C; HIV-negative participants also underwent a second round of HIV testing at FV1. As funds were available only for one round of hepatitis $B$ and $C$ testing, no tests were conducted at FV2. In this paper we present data from the FV1. Harm reduction services were initiated after completion of FV1.

Participants were recruited through peer-referral and targeted outreach by outreach workers (ORW). Prior to study initiation, a mapping exercise was conducted to identify all hotspots where PWIDs congregated in central, east, north-east and north-west districts of Delhi where the five Sahara Drop-in Centers (DIC) were located. The peer-referral recruitment process was initiated using eleven 'seed' participants (recruiters) across the five study sites; each 'seed' participant was provided with five recruitment coupons to give out to PWIDs in their network. New recruits were linked to the recruiter through unique ID numbers; each new recruit received five coupons to recruit other PWIDs and the recruiter received a 'food coupon' for each new PWID s/he brought into the study. Food coupons were exchanged for food at selected restaurant outlets. For recruitment using targeted outreach, ORWs visited hotspots to invite PWIDs to participate in the study and willing PWIDs were directed to the study site with an ORW coupon. All PWIDs who had a peer-referral recruitment coupon were considered recruited through peer-referral while those who came to the site with an ORW coupon were listed as recruited by ORWs. Walk-in clients who did not have either a peer-referral or ORW coupon were also permitted to enroll. All PWIDs who enrolled in the study, including walk-in clients and those recruited by ORWs, received five recruitment coupons each to recruit other PWIDs. Every hotspot was covered during the recruitment phase and recruitment continued till no new PWIDs could be found [21]. To be eligible, participants had to be 18 years of age or older, current IDU defined as injecting at least once in the last 3 months and residing in Delhi. All participants provided written informed consent. All participants received Rs 40 (approximately 80 US cents) for participating in the behavioral survey.

To detect duplicate registrations at recruitment, we used a web-based, live data-base accessible by all five DICs which allowed the DIC site manager to verify new clients against already registered clients. Registration procedures included the recording of photographs and 
the following identifiers: name, age, gender, marital status, height, weight and fore-arm length - participants presenting with similar identifiers were identified by the computer program for further scrutiny [21].

At FV1, ORWs contacted participants in the community to return for their follow-up visit. Returning participants completed FV1 data collection which included laboratory testing and a behavioral survey. As very few female PWIDs $(n=26)$ were recruited in the study, they have been excluded from the analysis.

The study was approved by the National AIDS Control Organization (NACO) Ethics Committee in Delhi, the Research Ethics Committee of PATH in Seattle, USA and the Institutional Review Board of the Population Council in New York. All participants provided written informed consent for participating in the behavioral survey and collection of blood samples.

\section{Laboratory testing}

HIV sero-status at baseline was determined using NACO HIV testing guidelines via rapid tests: HIVnegative status was based on a single highly sensitive rapid test (DetermineTM HIV-1/2; Inverness Medical Japan Co Ltd., Japan) and a positive result on two additional confirmatory rapid tests (SignalTM HIV-1/2; Span Diagnostics, Surat India and TridotTM; Biomed Industries, Himachal Pradesh, India). At FV1, all HIV-negative male PWIDs were tested using a fourth generation Antigen-Antibody test (ARCHITECT HIV Ag/Ab Combo; Abbott Laboratories, Illinois, USA) followed by a confirmatory Western Blot Assay (HIV Blot 2.2 Western blot assay; MP Biomedicals Asia Pacific Limited, Singapore). For participants with a negative or indeterminate result on the Western blot test at FV1, a plasma HIV RNA PCR test (COBAS ${ }^{\bullet}$ Ampliprep/COBAS $^{\circ}$ TaqMan $^{\circ}$ HIV-1 Test; Roche Diagnostics, Tokyo, Japan) was conducted on the FV1 plasma sample to identify any window period infection at FV1.

Hepatitis $\mathrm{C}$ antibodies were detected using CMIA/ Electrochemiluminescence test (ARCHITECT AntiHCV Reagent Kit; Abbott Laboratories, Illinois, USA). All participants with a positive $\mathrm{HCV}$ antibody test underwent $\mathrm{HCV}$ RNA PCR testing (COBAS ${ }^{\circ}$ AmpliPrep/COBAS ${ }^{\circ}$ TaqMan $^{\circ}$ HCV Tests; Roche Diagnostics, Tokyo, Japan); participants positive for $\mathrm{HCV}$ RNA were labelled HCV positive. We have considered $\mathrm{HCV}$ RNA positivity as the marker for $\mathrm{HCV}$ prevalence so as to accurately identify active $\mathrm{HCV}$ infection and, therefore, PWIDs who have the potential to transmit infection to others. HBV infection was detected using serum hepatitis B surface antigen test (ARCHITECT HBsAg Reagent Kit; Abbott Laboratories, Illinois, USA).

\section{Statistical analysis}

All quantitative analyses were conducted using STATA version 11.2 (Stata Corp, College Station, Texas, USA). Participants were categorized into four groups with regard to their HIV and HCV status: (a) Sero-negative (HIV-, HCV-), (b) HIV mono-infection (HIV+, HCV-), (c) HCV mono-infection (HIV-, $\mathrm{HCV}+$ ), and (d) HIV \& $\mathrm{HCV}$ co-infection (HIV+, $\mathrm{HCV}+)$. Comparisons were made between sero-negative participants (HIV- and HCV) and the different infection groups (HIV mono-infection, HCV mono-infection, HIV \& HCV co-infection) using Chi-squared test for categorical variables and MannWhitney $U$ test for continuous variables. Prevalence of $\mathrm{HBV}$ was reported in these four infection categories.

We used a multinomial logistic regression model to predict the relative risk ratios for HIV mono-infection, $\mathrm{HCV}$ mono-infection and HIV-HCV co-infection as compared to the sero-negative PWIDs (reference category). Variables that were found to be significant in at least one of the infection categories from the bivariate analysis from Table 1, were included in the regression model (Table 2). Regional origin was classified into three categories - Delhi, three adjacent states of Delhi (includes neighboring states Uttar Pradesh, Haryana and Rajasthan), and other states. HIV knowledge was assessed using a 6item index comprising knowledge that HIV transmission can be prevented by (i) correct and consistent use of condoms for sex and (ii) having a monogamous uninfected sexual partner; that (iii) sharing of needles/syringes increase the risk of HIV transmission, that (iv-v) HIV infection cannot spread from mosquito bites or from sharing food; and that (vi) healthy-looking people can be infected with HIV. Comprehensive knowledge was considered to be present if participants responded correctly on all 6 items. Prior knowledge of HIV status was also included.

Risky injection behavior was derived from a 5-item index of various injection practices in the last month:

(i) using needles or syringes previously used by someone else, (ii) back/ front loading/splitting of drugs, (iii) receiving an injection with a syringe filled by someone else, (iv) drawing up drugs from a common container, and (v) sharing vial, cooker, container, cotton, filter or water with other injecting partners. A regular sex partner was defined as a spouse or live-in partner. A paid partner was someone the respondent had sex with in exchange for money, gifts or drugs. A non-regular partner was someone the respondent was not living with or paid for sex. Safe sex was defined as use of condoms every time the respondent had sex. Unsafe sex was defined as inconsistent or no use of condoms.

\section{Results}

A total of 3792 male PWIDs were recruited at baseline [20]. Between baseline and FV1, 44 duplicate clients 
Table 1 Socio-demographic characteristics, risky injection and sexual behaviors and service utilization by male PWID by HIV, HCV and HIV-HCV co-infection status, Delhi (2012)

\begin{tabular}{|c|c|c|c|c|c|c|c|c|}
\hline & \multirow[t]{2}{*}{ Total } & \multirow{2}{*}{$\begin{array}{l}\text { Sero-negative } \\
\%(n)\end{array}$} & \multicolumn{2}{|c|}{ HIV mono-infected } & \multicolumn{2}{|c|}{ HCV mono-infected } & \multicolumn{2}{|c|}{ HIV \& HCV co-infected } \\
\hline & & & $\%(\mathrm{n})$ & $\overline{p \text {-value }}{ }^{1}$ & $\%(n)$ & $p$-value & $\%(n)$ & $p$-value ${ }^{1}$ \\
\hline Number of PWIDs & 2,292 & $100.0(916)$ & $100.0(146)$ & & $100.0(781)$ & & $100.0(449)$ & \\
\hline Prevalence of sero-infections (\%) & & 40.0 & 6.4 & & 34.1 & & 19.6 & \\
\hline \multicolumn{9}{|l|}{ Socio-demographic characteristics } \\
\hline Age (median, IQR) & $29(23,37)$ & $28(23,38.5)$ & $26(22,35)$ & $0.016^{2}$ & $29(24,38)$ & $0.873^{2}$ & $30(24,35)$ & $0.772^{2}$ \\
\hline Education & & & & 0.371 & & 0.972 & & \\
\hline Illiterate & $47.6(1090)$ & $46.8(429)$ & $48.6(71)$ & & $46.3(361)$ & & $51(229)$ & $2.8 ; 0.246$ \\
\hline Class 1-6 & $27.6(632)$ & $27.4(251)$ & $30.8(45)$ & & $27.6(215)$ & & $27(121)$ & \\
\hline 7 or higher & $24.8(569)$ & $25.8(236)$ & $20.6(30)$ & & $26.2(204)$ & & $22.1(99)$ & \\
\hline Marital status & & & & 0.112 & & 0.545 & & 0.043 \\
\hline Married/cohabiting & $34.7(796)$ & $36.7(336)$ & $30.1(44)$ & & $34.8(272)$ & & $32.1(144)$ & \\
\hline Never married & $52.9(1213)$ & $52.6(482)$ & $54.1(79)$ & & $53(414)$ & & $53(238)$ & \\
\hline Divorced/widowed & $12.4(283)$ & $10.7(98)$ & $15.8(23)$ & & $12.2(95)$ & & $14.9(67)$ & \\
\hline Religion & & & & 0.921 & & $<0.001$ & & 0.609 \\
\hline Hindu & $62(1418)$ & $58.5(535)$ & $58.9(86)$ & & $69.5(541)$ & & $57(256)$ & \\
\hline Non-Hindu & $38(870)$ & $41.5(380)$ & $41.1(60)$ & & $30.5(237)$ & & $43(193)$ & \\
\hline Regional origin & & & & 0.003 & & 0.010 & & 0.010 \\
\hline Delhi & $25.4(580)$ & $25.4(232)$ & $24.7(36)$ & & $25.4(198)$ & & $25.4(114)$ & \\
\hline 3 states adjacent to Delhi & $44.4(1016)$ & $39.9(365)$ & $53.4(78)$ & & $46.2(360)$ & & $47.4(213)$ & \\
\hline Others & $30.2(692)$ & $34.7(317)$ & $21.9(32)$ & & $28.4(221)$ & & $27.2(122)$ & \\
\hline Duration of residence in Delhi (median, IQR) & $22(15,30)$ & $22(15,30)$ & $20(12,29)$ & $0.142^{2}$ & $23(16,30)$ & $0.124^{2}$ & $23(15,30)$ & $0.208^{2}$ \\
\hline Accommodation & & & & 0.183 & & 0.879 & & 0.801 \\
\hline Living at home with family & 44 (1009) & $44.4(407)$ & $39.7(58)$ & & $44.4(347)$ & & $43.9(197)$ & \\
\hline Living In rented/paying guest & $17.5(400)$ & $17.6(161)$ & $14.4(21)$ & & $18.4(144)$ & & $16.5(74)$ & \\
\hline Living on the street & $38.5(883)$ & $38(348)$ & $45.9(67)$ & & $37.1(290)$ & & $39.6(178)$ & \\
\hline Comprehensive HIV knowledge & & & & 0.190 & & 0.908 & & 0.048 \\
\hline No/incomplete knowledge & $63.2(1448)$ & $64.5(591)$ & $58.9(86)$ & & $64.8(506)$ & & $59(265)$ & \\
\hline Complete knowledge & $36.8(844)$ & $35.5(325)$ & $41.1(60)$ & & $35.2(275)$ & & $41(184)$ & \\
\hline Alcohol use & & & & 0.046 & & 0.859 & & 0.009 \\
\hline Never & $54.4(1246)$ & $51.9(475)$ & $61(89)$ & & $53.1(414)$ & & $59.8(268)$ & \\
\hline 2 times/week or less & $35.6(815)$ & $37.1(339)$ & $33.6(49)$ & & $35.8(279)$ & & $33(148)$ & \\
\hline 3 times a week or more & $10(228)$ & $11(101)$ & $5.5(8)$ & & $11.2(87)$ & & $7.1(32)$ & \\
\hline \multicolumn{4}{|c|}{ Sex with regular or paid/non-regular female partner in last 3 months } & 0.008 & & 0.110 & & $<0.001$ \\
\hline No sex & $63.5(1455)$ & $57.6(528)$ & $71.2(104)$ & & $62.6(489)$ & & $74.4(334)$ & \\
\hline
\end{tabular}


Table 1 Socio-demographic characteristics, risky injection and sexual behaviors and service utilization by male PWID by HIV, HCV and HIV-HCV co-infection status, Delhi (2012) (Continued)

\begin{tabular}{|c|c|c|c|c|c|c|c|c|}
\hline Safe sex & $11(251)$ & $12.3(113)$ & $8.2(12)$ & & $10.5(82)$ & & $9.8(44)$ & \\
\hline Unsafe sex & $25.6(586)$ & $30(275)$ & $20.6(30)$ & & $26.9(210)$ & & $15.8(71)$ & \\
\hline \multicolumn{9}{|l|}{ Service utilization in the last 3 months } \\
\hline Accessed NSP services & & & & $<0.001$ & & $<0.001$ & & $<0.001$ \\
\hline No & $55.5(1271)$ & $70.4(645)$ & $43.2(63)$ & & $48.9(382)$ & & $40.3(181)$ & \\
\hline Yes & $44.6(1021)$ & $29.6(271)$ & $56.9(83)$ & & $51.1(399)$ & & $59.7(268)$ & \\
\hline Accessed detox/rehab services & & & & 0.696 & & 0.270 & & 0.148 \\
\hline No & $94.1(2157)$ & $93.3(855)$ & $92.5(135)$ & & $94.6(739)$ & & $95.3(428)$ & \\
\hline Yes & $5.9(135)$ & $6.7(61)$ & $7.5(11)$ & & $5.4(42)$ & & $4.7(21)$ & \\
\hline Accessed OST services & & & & 0.847 & & 0.643 & & 0.583 \\
\hline No & $89.3(2046)$ & $89.2(817)$ & $89.7(131)$ & & $89.9(702)$ & & $88.2(396)$ & \\
\hline Yes & $10.7(246)$ & $10.8(99)$ & $10.3(15)$ & & $10.1(79)$ & & $11.8(53)$ & \\
\hline \multicolumn{9}{|l|}{ Duration and frequency of injection drug use } \\
\hline Time since first injection & & & & $<0.001$ & & $<0.001$ & & $<0.001$ \\
\hline 1 year or less & $13.2(299)$ & $22.5(204)$ & $9.7(14)$ & & $8.6(66)$ & & $3.4(15)$ & \\
\hline $2-5$ years & $53.8(1221)$ & $52.7(478)$ & $54.5(79)$ & & $55.1(425)$ & & $53.6(239)$ & \\
\hline $6-10$ years & $21.6(490)$ & $15.9(144)$ & $27.6(40)$ & & $22.5(174)$ & & $29.6(132)$ & \\
\hline 11 years or more & $11.5(260)$ & $8.9(81)$ & $8.3(12)$ & & $13.9(107)$ & & $13.5(60)$ & \\
\hline Number of days injected in the past month & & & & $<0.001$ & & $<0.001$ & & $<0.001$ \\
\hline Not injected & $26.5(606)$ & $44.4(406)$ & $19.2(28)$ & & $15.7(122)$ & & $11.1(50)$ & \\
\hline $1-10$ days & $20.5(470)$ & $23.5(215)$ & $16.4(24)$ & & $18.2(142)$ & & $19.8(89)$ & \\
\hline $11-20$ days & $16.7(382)$ & $11.4(104)$ & $17.1(25)$ & & $21.2(165)$ & & $19.6(88)$ & \\
\hline 21-30 days & $36.3(830)$ & $20.7(189)$ & $47.3(69)$ & & $44.9(350)$ & & $49.4(222)$ & \\
\hline Risky injection behavior in the past 1 month & & & & $<0.001$ & & $<0.001$ & & $<0.001$ \\
\hline No risk & $47.6(1090)$ & $63.7(583)$ & $39(57)$ & & $37.6(294)$ & & $34.7(156)$ & \\
\hline At least one risky injection & $52.4(1202)$ & $36.4(333)$ & $61(89)$ & & $62.4(487)$ & & $65.3(293)$ & \\
\hline \multicolumn{9}{|l|}{ Prior HIV test } \\
\hline Prior knowledge of own HIV status & & & & 0.001 & & 0.778 & & $<0.001$ \\
\hline Know the result & $71.4(1631)$ & $74.5(681)$ & $61(89)$ & & $73.9(575)$ & & $64.1(286)$ & \\
\hline Not tested/ Result not known & $28.6(653)$ & $25.5(233)$ & $39(57)$ & & $26.1(203)$ & & $35.9(160)$ & \\
\hline
\end{tabular}

Chi-squared test, unless specified, for each infection category compared with sero-negative participants

${ }^{2}$ Mann-Whitney U test for each infection category compared with sero-negative participants

NSP: Needle Syringe Program; OST: Oral Substitution Therapy 
Table 2 Multinomial Logistic Regression analysis to determine predictors of HIV, HCV and HIV-HCV prevalence among Male PWID in Delhi $(2012)^{1,2}$

\begin{tabular}{|c|c|c|c|}
\hline & HIV mono-infection & HCV mono-infection & HIV \& HCV co-infection \\
\hline & RRR (95\% Cl); p-value & RRR (95\% Cl); p-value & RRR (95\% Cl); p-value \\
\hline \multicolumn{4}{|l|}{ Comprehensive HIV knowledge } \\
\hline No comprehensive knowledge & 1.00 & 1.00 & 1.00 \\
\hline Having comprehensive knowledge & $1.50(1.03-2.20) ; 0.037$ & $1.08(0.86-1.34) ; 0.523$ & $1.47(1.13-1.91) ; 0.004$ \\
\hline \multicolumn{4}{|l|}{ Alcohol use } \\
\hline Never & 1.00 & 1.00 & 1.00 \\
\hline$\leq 2$ times/week & $0.85(0.57-1.27) ; 0.432$ & $0.96(0.77-1.21) ; 0.759$ & $0.88(0.67-1.16) ; 0.363$ \\
\hline$\geq 3$ times a week & $0.62(0.28-1.36) ; 0.236$ & $1.27(0.89-1.81) ; 0.18$ & $0.85(0.53-1.36) ; 0.486$ \\
\hline \multicolumn{4}{|c|}{ Sex with any female partner in last 3 months ${ }^{3}$} \\
\hline No sex & 1.00 & 1.00 & 1.00 \\
\hline Safe sex & $0.55(0.29-1.06) ; 0.076$ & $0.79(0.56-1.11) ; 0.17$ & $0.62(0.41-0.95) ; 0.026$ \\
\hline Unsafe sex & $0.60(0.37-0.99) ; 0.046$ & $0.93(0.71-1.21) ; 0.567$ & $0.40(0.28-0.57) ;<0.001$ \\
\hline \multicolumn{4}{|l|}{ Accessed NSP services last 3 months } \\
\hline No & 1.00 & 1.00 & 1.00 \\
\hline Yes & $1.91(1.26-2.89) ; 0.002$ & $1.37(1.08-1.73) ; 0.009$ & $1.74(1.32-2.30) ;<0.001$ \\
\hline \multicolumn{4}{|l|}{ Duration of injecting drug use } \\
\hline$\leq 1$ year & 1.00 & 1.00 & 1.00 \\
\hline $2-5$ years & $1.90(1.02-3.51) ; 0.042$ & $2.13(1.53-2.95) ;<0.001$ & $5.14(2.91-9.09) ;<0.001$ \\
\hline $6-10$ years & $3.01(1.52-5.97) ; 0.002$ & $2.74(1.86-4.03) ;<0.001$ & $8.53(4.65-15.64) ;<0.001$ \\
\hline 11 years of more & $2.07(0.86-5.00) ; 0.106$ & $3.14(2.00-4.93) ;<0.001$ & $8.03(4.09-15.76) ;<0.001$ \\
\hline \multicolumn{4}{|c|}{ Frequency of injection during the past month } \\
\hline Never & 1.00 & 1.00 & 1.00 \\
\hline $1-10$ days & $0.93(0.47-1.83) ; 0.831$ & $1.61(1.14-2.29) ; 0.007$ & $2.26(1.43-3.57) ;<0.001$ \\
\hline $11-20$ days & $1.57(0.76-3.24) ; 0.226$ & $3.15(2.12-4.66) ;<0.001$ & $3.46(2.09-5.74) ;<0.001$ \\
\hline 21-30 days & $2.47(1.30-4.69) ; 0.006$ & $3.47(2.43-4.95) ;<0.001$ & $4.83(3.06-7.61) ;<0.001$ \\
\hline \multicolumn{4}{|l|}{ Risky injection practice in last one month } \\
\hline Never & 1.00 & 1.00 & 1.00 \\
\hline At least one risky injection & $1.40(0.87-2.26) ; 0.171$ & $1.39(1.06-1.83) ; 0.016$ & $1.38(1.01-1.89) ; 0.045$ \\
\hline \multicolumn{4}{|l|}{ Prior knowledge of own HIV status ${ }^{4}$} \\
\hline Know the result & 1.00 & 1.00 & 1.00 \\
\hline Not tested/Don't know the result & $1.92(1.31-2.82) ; 0.001$ & $0.97(0.76-1.23) ; 0.813$ & $1.65(1.25-2.17) ;<0.001$ \\
\hline
\end{tabular}

${ }^{1}$ Reference category of the dependent variable in the multinomial regression model is sero-negative participants $(n=916)$ compared to participants with HIV mono-infection $(n=146)$, HCV mono-infection $(n=781)$ and HIV \& HCV co-infection $(n=449)$

${ }^{2}$ Controlled for age, religion, marital status and regional origin

${ }^{3}$ Any female partner includes regular, non-regular or paid

${ }^{4}$ At baseline

were identified and removed from the database. Of the remaining male PWIDs $66.2 \%$ (2480/3748) participants returned for their FV1; the behavioral survey and blood tests were conducted for 2353 participants (Fig. 1). As shown in Fig. 1, HIV test results were available for 2348 participants, hepatitis B for 2312 participants and hepatitis C test results for 2294 participants. Our data analysis includes the 2292 male PWIDs for whom we have HIV, HBV and HCV test results.
The median age of study participants was 29 years (IQR: 23-37). Most of them were illiterate (48\%), never married (53 \%), and of the Hindu faith (62\%). All participants resided in Delhi, however, $44 \%$ were originally from the three adjacent states of Delhi and $33 \%$ from other states (Table 1). While most of the participants were home-based (44\%), a sizeable proportion lived on the streets (39\%). HIV knowledge was low (37\%); $54 \%$ reported never having consumed alcohol and $63 \%$ had been sexually inactive in the last 3 months. Only $9.1 \%$ 


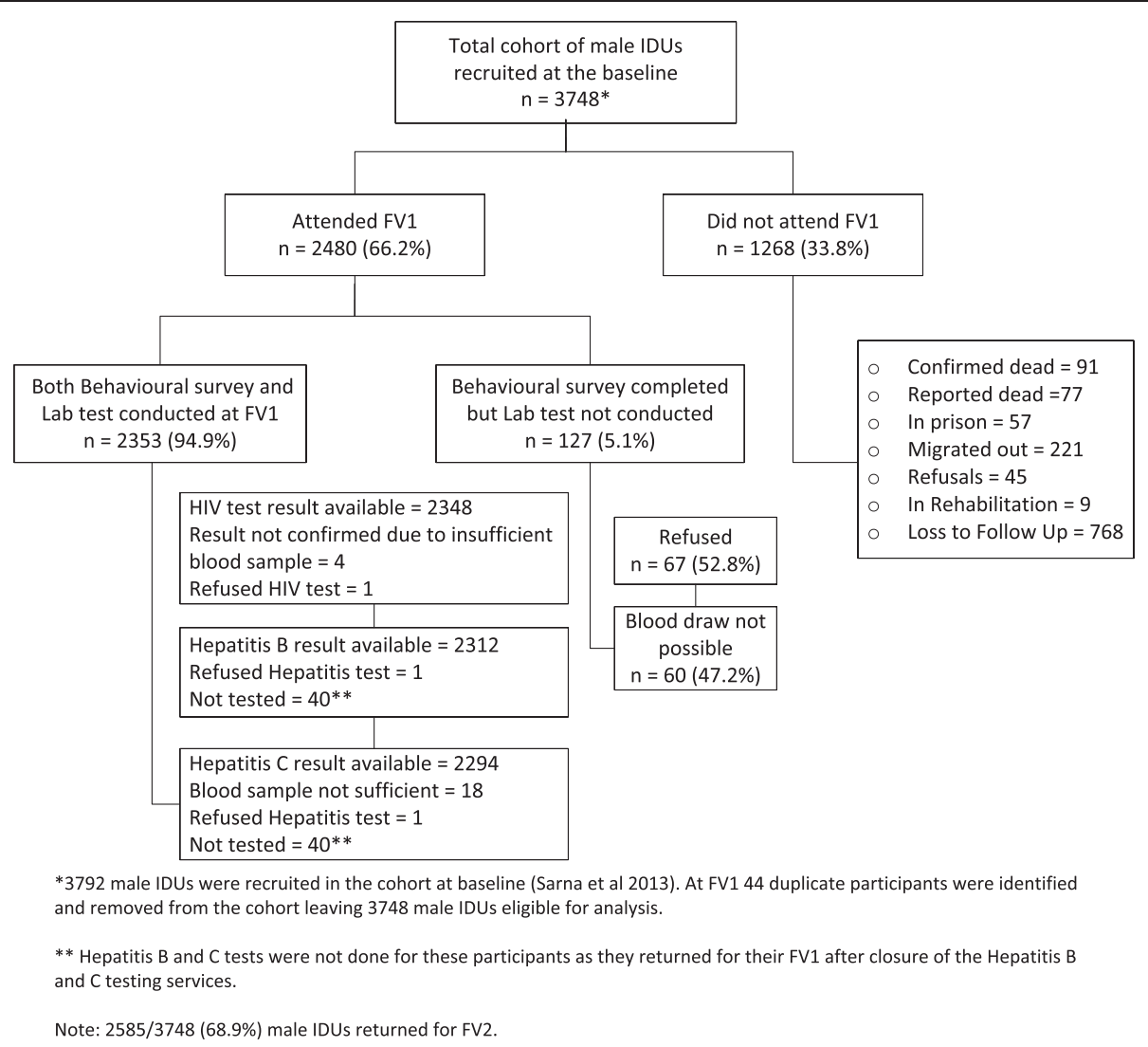

Fig. 1 Follow-up and Laboratory testing of Male PWIDs in the baseline full Cohort

of participants had ever heard of hepatitis B and $3.1 \%$ had heard of hepatitis $\mathrm{C}$ infections (data not shown). A third had been injecting for more than 6 years. Unsafe injection practices were common: $26.6 \%$ reported injecting with a used needle in the last one month, $32 \%$ had shared injecting equipment, $15.4 \%$ had received a prefilled injection from another PWID, 18.3 \% had injected drugs using front/ back loading and splitting and $48.1 \%$ had drawn drugs from a common container (data not shown); overall 52.4\% participants reported risky injection behavior in the last one month (Table 1). $44.5 \%$ had accessed needle/syringe exchange/distribution services in the last 3 months, while uptake of oral substitution therapy (OST) and rehabilitation services was low (Table 1). The vast majority of participants (69.2\%) reported having injected opioids (heroin/brown sugar/smack/synthetic opioids) and $73.4 \%$ had injected tranquilizers/antihistaminics in the last one month; a few reported injecting cocaine $(0.5 \%)$ and amphetamines (3.1\%). Participants typically injected a cocktail of two or three drugs consisting of one opioid and tranquilizers and/ or antihistaminics (data not shown).

\section{Prevalence of HIV, HCV and HIV-HCV co-infection}

HCV antibody tests were positive for $70.9 \%$ (1626/2294) of the participants; of those $75.7 \%(1230 / 1626)$ participants tested positive on HCV RNA PCR test, and were considered HCV positive; overall $53.7 \%(1230 / 2292)$ of participants were HCV RNA positive. Overall $6.4 \%(n=146)$ participants had HIV mono-infection, $34.1 \%(\mathrm{n}=781)$ had HCV mono-infection and $19.6 \%(\mathrm{n}=449) \mathrm{HIV}$ \& HCV co-infection while $39.9 \%$ were sero-negative for HIV or HCV. HIV prevalence was $25.9 \%$ (595/2292); this includes the 109 new seroconversions among HIV-negative PWIDs documented between baseline and FV1. Table 1 highlights the differences between the various infection groups compared to uninfected participants. On bivariate analysis, there were no significant differences between various infection groups and uninfected participants on age, education, duration of residence in Delhi or accommodation. All three infection groups were more likely to have migrated from the 3 states adjacent to Delhi. HIV \& HCV co-infected participants were more likely to have comprehensive HIV knowledge, report non-consumption of alcohol, be divorced/widowed and not sexually active in the last 3 months than seronegative participants. HIV mono-infected participants were also more likely to be not sexually active. All three infection groups were more likely to have been injecting for longer periods ( $>2$ years) and on more days every month, to have higher scores on the risky 
injection index and to have accessed NSP services than uninfected participants (Table 1).

\section{Prevalence of HBV, HIV-HBV and HBV-HCV co-infections}

Hepatitis B surface antigen was detected in $9.7 \%$ (222/ 2292) participants. HBV prevalence was $6.7 \%(52 / 781)$ among the HCV mono-infected and $8.7 \%(39 / 449)$ among the HIV-HCV co-infected participants. The highest HBV prevalence (26\%; 38/146) was found among those who were HIV positive but HCV negative. The prevalence of HBV mono-infection was $10.2 \%$ (93/916).

\section{Results from multivariate multinomial logistic regression}

Results of multinomial logistic regression analysis to determine correlates of HIV mono-infection, HCV monoinfection, and HIV \& HCV co-infection with reference to uninfected participants, after controlling for age, marital status, religion and regional origin, are presented in Table 2. Participants who had not undertaken HIV testing or did not know of their HIV-status prior to the study were at higher risk of HIV mono-infection (RRR: 1.92) and HIV-HCV co-infection (RRR: 1.65). Longer duration of drug injections was associated with a higher likelihood of HCV mono-infection with a dose response effect $(2-5$ years RRR: 2.13 ; $6-10$ years RRR: $2.74 ;>11$ years RRR: 3.14 years) and HIV-HCV co-infection $(2-5$ years RRR: $5.14 ; 6-10$ years RRR: 8.53 ; $>11$ years RRR: 8.03$)$. A higher frequency of injection days/month was associated with a higher likelihood, with a dose-response effect, for HCV mono-infection ( $\leq 10$ days/month RRR: $1.61 ; 11-20$ days/month RRR: 3.15 ; $21-30$ days/month RRR: 3.47$)$ and HIV-HCV co-infections ( $\leq 10$ days/month RRR: 2.26 ; $11-20$ days/month RRR: 3.46; 21-30 days/month RRR: 4.83). Risky injection behavior in the last one month was an independent predictor of HCV mono-infection (RRR: 1.39) and HIV-HCV co-infection (RRR: 1.38).

Having comprehensive HIV knowledge was associated with a higher risk of HIV mono-infection (RRR: 1.5) and HIV-HCV co-infection (RRR: 1.47). Further, a higher likelihood of all three categories of infection was observed among participants who had accessed needle syringe program services in the last three months (HIV mono-infection RRR: 1.91; HCV mono-infection RRR: 1.37; HIV-HCV co-infection RRR: 1.74). These results are unexpected and need to be interpreted with caution. On further analysis, it was observed that (a) comprehensive HIV knowledge increased with longer duration of injection use ( $<1$ year: $27 \%$; $2-5$ years: $36 \%$; $6-10$ years: $41 \%$; >11 years: $45 \%$; p < 0.001); (b) the use of needle syringe program services increased with longer duration of injection drug use ( $<1$ year: $27 \%$; $2-5$ years: $44 \%$; $6-$ 10 years: $54 \%$; $>11$ years: $53 \%$; $<<0.001$ ) and frequency of injections in the last 1 month (no injection: $8 \%$; $1-10$ days: $48 \%$; 11-20 days: $63 \%$; 21-30 days: $62 \%$; $<<$
0.001); and (c) among PWIDs who accessed NSP services, risky injection practices increased with greater frequency of injections in last 1 month (no injections: $1.92 \%$; 1-10 days: $65.9 \%$; $11-20$ days: $76.9 \%$; $21-30$ days: $79.1 \%$; $\mathrm{p}<$ 0.001). However, comprehensive HIV knowledge was not associated with use of needle syringe programs services: $44 \%$ of participants with comprehensive knowledge and $46 \%$ without comprehensive knowledge had accessed needle syringe program services in the last 3 months $(\mathrm{p}=$ 0.26). Public sector HIV prevention services delivered through Targeted Intervention (TI) programs for PWIDs in Delhi have been in existence for several years. PWIDs with longer duration of injections are more likely to be a part of the PWID community for a longer period of time, and therefore, more likely to be covered by NSP and IEC services. PWIDs with a higher frequency of injections are more likely to seek NSP services due to a greater need for clean needles. Therefore, PWIDs with longer duration of injecting and greater frequency of injections remain most at risk for new infections despite accessing NSP services as they may not obtain a sufficient supply of clean needles to meet their need, and continue to engage in unsafe injection practices as shown. Needle syringe distribution as part of our study was initiated only after completion of FV1.

Any sex (safe sex RRR: 0.62 and unsafe sex RRR: 0.40) compared to no sex was associated with a lower likelihood of HIV-HCV co-infection; unsafe sex (RRR: 0.60) was also associated with a lower likelihood of HIV mono-infection. In our cohort, only $37 \%$ of the participants were sexually active; sexual activity decreased with duration of injection drug use ( $\leq 1$ year: $45 \% ; 2-5$ years: $36 \%$; $6-10$ years: $36 \%$; $\geq 11$ years: $35 \% ; p=0.052)$ and frequency of injections in the last 1 month (no injection: $41 \%$; $1-10$ days: $41 \%$; $11-20$ days: $35 \%$; $21-30$ days: $32 \%$; $<0.004)$. Thus, the risk of HIV and HCV infection in our cohort was mainly concentrated around injection behaviors and not sexual behavior. Among the sexually active PWIDs, 70 \% (586/837) reported unsafe sex in the last 3 months. Unsafe sex among PWIDs remains a key programmatic concern.

\section{Discussion}

In this paper, we report a high prevalence of HIV infection (25.9\%), hepatitis C infection (53.7\%), and more significantly, high HIV-HCV co-infection (19.6 \%) among male PWIDs in Delhi in 2012. HBV prevalence was lower at $9.7 \%$; while HIV-HBV co-infection was $3.4 \%$ overall, among HIV positive PWIDs without $\mathrm{HCV}$ the prevalence was $26 \%$. Our findings are at variance with a study conducted among PWIDs in Delhi in 2003 [16], which reported a prevalence of $37 \%$ for HIV, $36 \%$ for $\mathrm{HCV}$ infection, $\mathrm{HIV}-\mathrm{HCV}$ co-infection of $9.6 \%$ and $40 \%$ prevalence for HBV. The annual HIV Sentinel 
Surveillance (HSS) [22] has documented a change in HIV prevalence among PWIDs in Delhi from 14.4\% in 2003, $10 \%$ in 2006 to $18.2 \%$ in $2010-11$. The HSS is an annual exercise to monitor trends in HIV prevalence; HIV testing is conducted among 250 PWIDs, recruited through snowball sampling at two DICs in Delhi. One DIC overlaps geographically with one of our study sites. Our HIV, HBV and HCV prevalence are similar to those reported more recently from Chennai [9] with a HIV prevalence of $29.8 \%$, HCV prevalence of $62.1 \%$ and $11.1 \%$ for $\mathrm{HBV}$; and a recent study from Delhi that reports a HIV prevalence of $13.8 \%$; HCV mono-infection of $31 \%$ and HIV-HCV co-infection of $14.5 \%$ [23]. Another recent study from Punjab reports a similarly high prevalence of HIV (29\%), HCV prevalence of (49\%) and HIV-HCV co-infection $25.7 \%$ among PWIDs [18].

Existing reports about $\mathrm{HCV}$ prevalence are based predominantly on serological testing for anti HCV antibodies, and a positive result can represent an acute, chronic or resolved HCV infection [8, 16, 18, 23-26]. We report the prevalence of active $\mathrm{HCV}$ infection based on HCV RNA PCR testing; to the best of our knowledge this is the first study in the country to provide these estimates for a large cohort of PWIDs. This has relevance for the national program as only PWIDs with active HCV infection need treatment. Our finding that a quarter of the PWID with a positive HCV antibody test were negative for HCV RNA, suggests that existing studies, which predominantly used antibody test to report HCV prevalence, overestimate infections substantially.

Several studies report a higher HCV prevalence among PWIDs than HIV prevalence $[9,18,25,26]$. HCV is estimated to be 10 times more infectious than HIV, per unit of blood required for transmission [27] and recent evidence showing that $\mathrm{HCV}$ can remain infectious, after drying at room temperature, for up to six weeks in blood spots [28], may provide a biological explanation for the continuing spread of $\mathrm{HCV}$ among PWIDs as they continue to practice unsafe injection practices that include sharing equipment, drawing from a common container and injecting with used needles.

HBV prevalence was lower overall (9.7\%). The prevalence among the HIV positive PWIDs (12.8\%), including those with HCV infection, was similar to the $12 \%$ reported by Solomon et al., from Chennai [8]. However, it is noteworthy that HBV prevalence among HIV-positive participants, in the absence of $\mathrm{HCV}$, was $26 \%$. A study conducted in Manipur among HIV-positive PWIDs detected HB core antibodies, a marker of prior infection, in all their participants implying that all had been exposed to $\mathrm{HBV}$ in the past [14]. Most studies have used HBsAg as the marker of $\mathrm{HBV}[8,16]$; it is possible that in the presence of a high prevalence of active $\mathrm{HCV}$ infection, all $\mathrm{HBV}$ infections may not be detected using HBs antigen testing. HBsAg is a marker of acute infection that persists during chronic infection. However, in some patients with acute infection (1-5\%) and in the rare chronic low-level carrier, levels of HBsAg may be too low to be detected by standard assays and other tests may be required [29]. It is well documented that in dual infections, $\mathrm{HBV}$ and $\mathrm{HCV}$ interact, and affect immune responses. Available evidence demonstrates that the two viruses can inhibit each other simultaneously; the chronology of infection has a role in determining the dominant virus; and $\mathrm{HBV}$ and HCV can alternate their dominance [30-32]. However, the overall dominant effect in published literature appears to be HCV suppression of HBV. Furthermore, patients with chronic $\mathrm{HCV}$ may have occult or silent $\mathrm{HBV}$ infection with low levels of circulating HBV DNA, and lack the HBsAg and HBeAg markers and their antibodies $[30,33]$. Undetected HBV is a concern as the virus can be transmitted through percutaneous, sexual and mother-to-child routes. Future studies should consider using a wider panel of tests to determine HBV prevalence in this high risk population, as a one-time HBsAg test may underestimate prevalence.

As HBV and HCV co-infected patients have a higher risk of progressing to fulminant hepatitis, developing chronic infection, cirrhosis and hepatocellular carcinoma $[2,11]$, it is extremely important for targeted intervention programs for PWIDs to include HBV and HCV in prevention messages and counselling protocols for PWIDs and their families. TI programs should include $\mathrm{HBV}$ testing and vaccination, an effective prevention intervention. As there is no vaccine available for $\mathrm{HCV}$ and it may be a while before treatment becomes affordable and available through public health programs, the focus of primary prevention efforts should continue to be on safe injection practices in this population.

Unsafe injection practices, duration of injection drug use and frequency of injections were the main predictors for $\mathrm{HCV}$ mono-infection and HIV-HCV co-infection. Accessing NSP services in the last 3 months was associated with a higher risk of HIV mono-, HCV mono- and HIV-HCV co-infections and having comprehensive HIV knowledge was associated with higher risk of HIV-HCV co-infection. These findings must be interpreted with caution. Further analysis showed that the use of NSP services and having HIV knowledge increased with longer duration of injection drug use, and higher frequency of injections. It is very likely that PWIDs who had been injecting for longer periods and were injecting more frequently were more aware of harm reduction programs in the area, had a greater need for needles/syringes and had been exposed to IEC activities in the area, and therefore, were accessing NSP services. At the same time, more than half the participants continued unsafe injection practices in the last one month, especially, 
those who injected more frequently and accessed NSP services. It is possible that they are unable to obtain a sufficient supply of needles to meet their need. Thus, the main risk of infection remains rooted in unsafe injection practices, longer duration of injection drug use and more frequent injections. In our baseline findings, HIV positive participants reported higher risk behaviors and accessed NSP services more often [20]. As this is a prevalence study we cannot comment on when the infections occurred; thus, it is also very likely that among long duration PWIDs the infections may have occurred before participants started accessing NSP services. The majority of our participants were not sexually active (63\% overall and $74.3 \%$ among HIV-HCV co-infected participants) and sexual activity was not associated with increased HIV, HCV or HIV-HCV co-infection. However, among PWIDs who were sexually active, $70 \%$ reported unsafe sex in the last 3 months, which remains an important concern for the HIV prevention program.

More than half our study participants had never consumed alcohol. This is in contrast to findings from a 2012 study on drug use patterns across 11 states that reports the majority of PWIDs ever used alcohol in their lifetime [34]. It is possible that our study participants transitioned to injection drug use from other addictions without consuming alcohol. In our previous study with 800 PWID in Delhi, only 23 \% reported alcohol consumption [19]. Overall alcohol consumption in the general population in Delhi is low - $33.1 \%$ of men 15-49 years of age interviewed in NFHS-3 reported consuming alcohol [35].

The study is not without limitations. Just two-thirds (66.2\%) of our cohort returned for this follow up visit; while this does not impact the reporting of prevalence for HIV, HBV and HCV infections, the program would have benefitted from prevalence reported from the larger cohort. We used only HBsAg testing to detect HBV infection. Using a wider panel of tests may have allowed us to detect occult HBV infections or past exposures; this may be considered for future studies. Further, we would have benefited from follow-up HBV and HCV testing at FV2, in this cohort of PWIDs, to obtain incidence rates that would have provided important information for the program; unfortunately we could not do so due to resource constraints. Despite using multiple strategies to reach female PWIDs including female seeds for peer-referral and female out-reach workers, we were able to recruit only 26 female PWIDs. In our previous study in 2006, we used respondent-driven sampling and were able to only recruit 17 female PWIDs [19]. It is very likely that there are few female PWIDs in Delhi. This finding has also been reported from other parts of India and in Asian countries $[9,36]$. Anecdotal information from ORWs in Delhi suggests that non-injecting drug-use is more frequent among female drug users; further research with female non-injecting drug users is needed to better understand drug use in this population.

\section{Conclusion}

In conclusion, we report a high prevalence of $\mathrm{HIV}, \mathrm{HCV}$ and HIV-HCV co-infection among male PWIDs in Delhi. The prevalence of HBV, although lower than $\mathrm{HCV}$, is still high with nearly a tenth of the PWIDs carrying the virus. The PWID population continues to engage in unsafe injection practices and not access NSP services regularly. Targeted intervention programs need to urgently address this problem by including hepatitis $\mathrm{B}$ and $\mathrm{C}$ in HIV prevention messages and counseling, expand services to include hepatitis testing, and offer hepatitis B vaccination for HBV negative PWIDs. A push by governments and activists to reduce the cost of treatment for hepatitis $C$ is also needed to widen access to treatment for infected persons.

\section{Abbreviations}

PWID: People Who Inject Drugs; HIV: Human Immunodeficiency Virus; HBV: Hepatitis B; HCV: Hepatitis C; NACO: National AIDS Control Organization (of India); NSP: Needle/syringe Program; OST: Oral Substitution Therapy; RRR: Relative risk ratio; Cl: Confidence interval; IQR: Inter-quartile range.

\section{Competing interests}

We have no competing interest to report.

Support for this project was provided by the Arise Program - Enhancing HIV Prevention Programs for At-Risk populations, through financial support provided by the Canadian Government through Department of Foreign Affairs, Trade and Development Canada (DFATD), and via financial and technical support provided by PATH. Arise implements innovative HIV prevention initiatives for vulnerable communities, with a focus on determining cost-effectiveness through rigorous evaluations. The views expressed by the authors do not necessarily reflect the views of PATH, the Canadian Government, or DFATD. Financial support for HCV testing was provided by MSD (A subsidiary of Merck \& Co, Inc. USA).

\section{Authors' contributions}

LRS prepared the first draft of the manuscript, did the statistical analysis and contributed in conceptualizing the paper. AS designed the study, conceptualized the paper, gave critical comments on the first draft and prepared the final version of the paper. MPS contributed in data analysis, and writing and editing of the paper. VS and IM contributed in data interpretation. IT and JP reviewed the manuscript and provided technical inputs. WT contributed in conceptualization, data analysis and preparing the draft. All authors read and approved the final manuscript.

\section{Acknowledgments}

We would like to thank Dr Neeraj Dhingra, Deputy Director General, NACO, Mr JK Misra, Joint Director Targeted Interventions and Delhi State AIDS Control Society for their support to the project.

We would like to thank Sahara's team of dedicated staff without whom this project would be impossible to implement. All laboratory testing was undertaken by Super Religare Laboratories, India. And finally, our deepest thanks to the study participants for their willingness to share this information with us.

\section{Author details}

'Population Council, Zone 5A, India Habitat Centre, New Delhi 110003, India. ${ }^{2}$ Sahara Center for Residential Care and Rehabilitation, New Delhi, India. ${ }^{3}$ ARISE Project, PATH, Washington, DC, USA. ${ }^{4}$ Population Council, Washington, DC, USA.

Received: 19 November 2014 Accepted: 30 June 2015

Published online: 30 July 2015 


\section{References}

1. Crofts N, Dore G, Locarnini (Eds.) S. Hepatitis C: an Australian perspective. IP Communications: East Hawthorn; 2001.

2. Mehta SH, Vogt SL, Srikrishnan AK, Vasudevan CK, Murugavel KG, Saravanan $S$, et al. Epidemiology of hepatitis $C$ virus infection \& liver disease among injection drug users (IDUs) in Chennai, India. Indian J Med Res. 2010;132:706-14

3. Poynard T, Bedossa P, Opolon P. Natural history of liver fibrosis progression in patients with chronic hepatitis C. The OBSVIRC, METAVIR, CLINIVIR, and DOSVIRC groups. Lancet. 1997;349(9055):825-32.

4. Dore GJ, Freeman AJ, Law M, Kaldor JM. Is severe liver disease a common outcome for people with chronic hepatitis C? J Gastroenterol Hepatol. 2002;17(4):423-30

5. Bica I, McGovern B, Dhar R, Stone D, McGowan K, Scheib R, et al. Increasing mortality due to end-stage liver disease in patients with human immunodeficiency virus infection. Clin Infect Dis. 2001;32(3):492-7.

6. Silver D, Karnik G, Osinusi A, Silk R, Stabinski L, Doonquah L, et al. Effect of $\mathrm{HIV}$ on liver fibrosis among HCV-infected African Americans. Clin Infect Dis. 2013:56(9):1280-3.

7. Puoti M, Spinetti A, Ghezzi A, Donato F, Zaltron S, Putzolu V, et al. Mortality for liver disease in patients with HIV infection: a cohort study. J Acquir Immune Defic Syndr. 2000;24(3):211-7.

8. Solomon SS, Hawcroft CS, Narasimhan P, Subbaraman R, Srikrishnan AK, Cecelia AJ, et al. Comorbidities among HIV-infected injection drug users in Chennai. India Indian J Med Res. 2008;127(5):447-52.

9. Solomon SS, Srikrishnan AK, Mehta SH, Vasudevan CK, Murugavel KG,

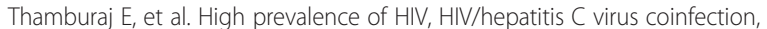
and risk behaviors among injection drug users in Chennai, India: a cause for concern. J Acquir Immune Defic Syndr. 2008;49(3):327-32.

10. Te HS, Jensen DM. Epidemiology of hepatitis B and C viruses: a global overview. Clin Liver Dis. 2010;14(1):1-21. vii.

11. Perz JF, Armstrong GL, Farrington LA, Hutin YJ, Bell BP. The contributions of hepatitis $B$ virus and hepatitis $C$ virus infections to cirrhosis and primary liver cancer worldwide. J Hepatol. 2006:45(4):529-38.

12. NACO. Annual Report 2012-13. New Delhi: National AIDS Control Organization, Department of AIDS Control, Ministry of Health and Family Welfare, Government of India; 2013.

13. Mahanta J, Medhi GK, Paranjape RS, Roy N, Kohli A, Akoijam BS, et al. Injecting and sexual risk behaviours, sexually transmitted infections and HIV prevalence in injecting drug users in three states in India. AIDS 2008;22(5):S59-68.

14. Saha MK, Chakrabarti S, Panda S, Naik TN, Manna B, Chatterjee A, et al. Prevalence of HCV \& HBV infection amongst HIV seropositive intravenous drug users \& their non-injecting wives in Manipur, India. Indian J Med Res. 2000;111:37-9.

15. Devi Kh S, Brajachand N, Singh HL, Singh YM. Co-infection by human immuno deficiency virus, hepatitis $B$ and hepatitis $C$ virus in injecting drug users. J Commun Dis. 2005;37(1):73-7.

16. Baveja UK, Chattopadhya D, Khera R, Joshi PM. A cross sectional serological study of the co-infection of hepatitis B virus, hepatitis $C$ virus and human immunodeficiency virus amongst a cohort of IDUs at Delhi. Indian J Med Microbiol. 2003;21(4):280-3.

17. Basu D, Kumar V, Sharma AK, Barnwal PK, Mattoo SK. Seroprevalence of antihepatitis $\mathrm{C}$ virus (anti-HCV) antibody and HCV-related risk in injecting drug users in northern India: Comparison with non-injecting drug users. Asian Journal of Psychiatry. 2013;6(1):52-5

18. Panda S, Roy T, Pahari S, Mehraa J, Sharma N, Singh G, et al. Alarming epidemics of human immunodeficiency virus and hepatitis $C$ virus among injection drug users in the northwestern bordering state of Punjab, India: prevalence and correlates. Int J STD AIDS, 2013;25(8):596-606.

19. Sarna A, Tun W, Bhattacharya A, Lewis D, Singh YS, Apicella L. Assessment of unsafe injection practices and sexual behaviors among male injecting drug users in two urban cities of India using respondent driven sampling. Southeast Asian J Trop Med Public Health. 2012;43(3):652-67.

20. Sarna A, Tun W, Sharma V, Sebastian M, Madan I, Yadav A, et al. High uptake of HIV testing in a cohort of male injection drug users in Delhi, India: prevalence and correlates of HIV infection. AIDS Behav. 2013;17(7):2479-89.

21. Tun W, Sebastian MP, Sharma V, Madan I, Souidi S, Lewis D, et al. Strategies for recruiting injection drug users for HIV prevention services in Delhi. India Harm Reduct J. 2013;10(1):16
22. NACO. HIV Sentinel Surveillance 2010-11: A Technical Brief. : National AIDS Control Organization, Department of AIDS Control, Ministry of Health and Family Welfare, Government of India; 2012.

23. Solomon S, Mehta S, Srikrishnan A, Solomon S, McFall A, Laeyendecker O, et al. Burden of hepatitis $C$ virus disease and access to hepatitis $C$ virus services in people who inject drugs in India: a cross-sectional study. Lancet Infectious Disease. 2015;15(1):36-45

24. Nelson PK, Mathers BM, Cowie B, Hagan H, Des Jarlais D, Horyniak D, et al. Global epidemiology of hepatitis $B$ and hepatitis $C$ in people who inject drugs: results of systematic reviews. Lancet. 2011;378(9791):571-83.

25. Mahanta J, Borkakoty B, Das HK, Chelleng PK. The risk of HIV and HCV infections among injection drug users in northeast India. AIDS Care. 2009:21(11):1420-4.

26. Chelleng PK, Borkakoty BJ, Chetia M, Das HK, Mahanta J. Risk of hepatitis C infection among injection drug users in Mizoram. India Indian J Med Res. 2008;128(5):640-6.

27. Crofts N. Going where the epidemic is. Epidemiology and control of hepatitis C among injecting drug users. Aust Fam Physician. 2001;30(5):420-5.

28. Paintsil E, Binka M, Patel A, Lindenbach BD, Heimer R. Hepatitis C Virus Maintains Infectivity for Weeks After Drying on Inanimate Surfaces at Room Temperature: Implications for Risks of Transmission. J Infect Dis 2014;209(8):1205-11.

29. Braunwald E, Fauci AS, Kasper DL, Hauser SL, Longo DL, Jameson JL. Harrison's Principles of Internal Medicine, USA. 15th Edition. Volume 2. McGraw-Hill Professional Publishing; 2001.

30. Ibarra RT. Treatment of HBV-HCV coinfection. Annals of Hepatology. 2006;5 Suppl 1:S49-52.

31. Fan $C L$, Wei $L$, Jiang $D$, Chen HS, Gao Y, Li RB, et al. Clinical and virological course of dual infection by hepatitis $B$ and $C$ viruses in China. Zhonghua Yi Xue Za Zhi. 2003;83(14):1214-8.

32. Sterling RK, Sulkowski MS. Hepatitis C virus in the setting of HIV or hepatitis B virus coinfection. Semin Liver Dis. 2004;24(Suppl 2):61-8.

33. Liu Z, Hou J. Hepatitis B virus (HBV) and hepatitis C virus (HCV) dual infection. Int J Med Sci. 2006;3(2):57-62.

34. Ambekar A. Association of Drug Use Pattern with Vulnerability and Service Uptakeamong Injecting Drug Users. Regional Office for South Asia, India: National AIDS Control Organization and United Nations Office of Drugs and Crime; 2012

35. International Institute for Population Sciences (IIPS) and Macro International. National Family Health Survey (NFHS-3), 2005-06. Report. IPS, Mumbai, India. 2007.

36. Wattana W, Griensven F, Rhucharoenpornpanich O, Manopaiboon C, Thienkrua W, Bannatham R, et al. Respondent driven sampling to assess characteristics and estimate the number of injection drug users in Bangkok, Thailand. Drug and Alcohol Dependence. 2007:90:228-33.

\section{Submit your next manuscript to BioMed Central and take full advantage of:}

- Convenient online submission

- Thorough peer review

- No space constraints or color figure charges

- Immediate publication on acceptance

- Inclusion in PubMed, CAS, Scopus and Google Scholar

- Research which is freely available for redistribution 